105 blood cultures, 2 were positive (1.9\%). One was positive for Streptococcus pneumonia and the other for Coagulase Negative Staphylococcus. The latter was concluded to be a contaminant of no clinical significance. As such only 1/105 was a true positive $(0.95 \%)$ and was fully sensitive to empirical therapy per our hospital antibiotic policy.

$34 / 105(32.3 \%)$ had positive respiratory virology, predominantly from nasopharyngeal aspirate. 34/105 (32.3\%) were discharged less than 48 hours from the time of the blood culture. Only two discharge letters specifically made reference to 'sepsis' as part of the diagnosis.

Conclusion Recent evidence to suggest the lack of clinical utility of blood cultures is supported by our local data. Indeed, approximately a third of our patients were discharged before the result of their blood culture was even known. The practice of sending blood cultures for these patients may be borne out of pragmatism around blood-taking from young children; however it is increasingly evident that they add little to clinical management in children with pneumonia.

\section{G340(P) ALICE: ATRAUMATIC LIMP IN CHILDREN PRESENTING TO THE EMERGENCY DEPARTMENT}

K O'Halloran, S Hurley, I Okafor, N Kandamany, R McNamara, P Fitzpatrick.

\subsection{6/archdischild-2018-rcpch.330}

Aims Atraumatic limp represents a significant proportion of presentations to the ED (1). The majority have self-resolving aetiologies. As part of a quality improvement project, we aimed to reduce unnecessary blood tests, imaging and follow up appointments, by implementing a guideline for the assessment of atraumatic limp.

Methods Following staff education and an awareness campaign, the guideline was implemented and prospectively audited. Data was collected from a clinical report form, and from healthcare records. The analysed data from March to April 2017 was compared to a previous epidemiological study, conducted in the same ED in 2015 (table 1).

\begin{tabular}{lll} 
Abstract G340(P) Table 1 & & \\
\hline Total Presentations with atraumatic limp & 2015 & 2017 \\
\hline Diagnosis of Transient Synovitis & 131 & 123 \\
Patients who had x-rays & $42 \%$ & $65 \%$ \\
Patients who had blood tests & $74 \%$ & $57 \%$ \\
Admissions & $28 \%$ & $33 \%$ \\
Follow up appointments & $3 \%$ & $6 \%$ \\
Representations with subsequent clinically significant diagnosis & $70 \%$ & $31 \%$ \\
change & $n=2$ & $n=2$ \\
(where no initial investigations) & & \\
\hline
\end{tabular}

Results 35 patients (40\% of total) were in a 'low risk group' (ie symptoms $<72$ hours, afebrile and a normal clinical exam). $14(40 \%)$ of these patients underwent investigations. None of these investigations changed the diagnosis. In total there were 5 changes in diagnosis post discharge. Two were following $\mathrm{x}$ rays reports with abnormalities. Three patients had a diagnosis change that were not initially investigated, one was subsequently diagnosed with HSP after representing with a lower limb non-blanching rash, one represented with ankle cellulitis and one was re-examined and referred to General Paediatrics with increased tone unilaterally. $60 \%$ of patients with a diagnosis of transient synovitis (TS) were discharged home without investigation. Of those diagnosed with TS $17 \%$ had bloods and $32 \%$ had an x-ray. $35 \%$ of these investigations were unnecessary according to the guideline.

Conclusions Implementation of a guideline can aid in the assessment of atraumatic limp and reduce the number of investigations and follow up appointments, without significant adverse outcome. Compliance with the guideline can be further improved.

\section{G341(P) QUALITY IMPROVEMENT PROJECT TO STREAMLINE ACUTELY UNWELL CHILDREN IN A DISTRICT GENERAL HOSPITAL (DGH) EMERGENCY DEPARTMENT}

1J Palman, ${ }^{1} \mathrm{~F}$ Miles, ${ }^{2} \mathrm{C}$ Emordi, ${ }^{1} \mathrm{~F}$ Lodge, ${ }^{1} \mathrm{~V}$ Stone, ${ }^{1} \mathrm{~F}$ Hikmet, ${ }^{1} \mathrm{~A}$ Nuti. ${ }^{1}$ General Paediatrics, Princess Alexandra Hospital NHS Trust, Harlow, UK; ${ }^{2}$ Accident and Emergency Department, Princess Alexandra Hospital NHS Trust, Harlow, UK

\subsection{6/archdischild-2018-rcpch.331}

Aims To develop a streamlined process ensuring severely unwell patients are triaged and referred directly to a specialist paediatrician within a timely fashion so that stabilisation and transfer to the ward occurs within the designated 4 hour emergency period. This quality improvement project aims to reduce the number of $>4$ hour patient waiting times by improving triage within the Paediatric Emergency Department (PED) and enhancing communication between the emergency and paediatric teams. This is important for DGHs with no paediatric assessment unit to monitor patients before admitting or discharging from hospital.

Methods An adapted version of the National Institute of Clinical Excellence (NICE) guidance for fever in the under 5 years (http://guidance.nice.org.uk/CG1) was created with specific thresholds to grade severity. The pathway implemented in this project, triaged high-risk patients to the paediatric team and low risk patients to the emergency team despite the method of referral. Data was prospectively collected over a 3 week period, analysed and presented in a departmental meeting and recommendations implemented for further cycles. We performed two cycles of quality improvement in this project. Results A total of 87 and 188 patients were included in cycle 1 (C1) and cycle 2 (C2) respectively. The demographics for each cycle were similar, the average age being 1.83 and 1.48 years respectively $(p=0.51)$. There was no statistical difference between the presenting complaints for each of the cycles $(p=0.969)$. Average PED duration was reduced from $3: 24$ hours to $2: 36$ hours $(p=0.0009)$. The number patients waiting $>4$ hours significantly reduced from C1: 17/87 (19.5\%) compared to C2: $14 / 188$ (6.9\%). In C2 we investigated the outcomes of PED attendance and discharge destination. During C2, 141/188 (75\%) patients were discharged, 26/ 188 (13.8\%) patients were admitted by the paediatric team, the remaining were ambulated or referred to another specialty. Conclusion By introducing our adapted NICE guidance pathway we demonstrated that average time spent in PED and number of children waiting $>4$ hours can be significantly 
reduced. Particularly unwell children were identified earlier and appropriately referred to the paediatricians from triage. Further cycles are underway to assess the impact of more senior cover in PED and improve the referral pathways.

\section{G342(P) GETTING BETTER IN THE FIGHT AGAINST COMMUNITY VIOLENCE}

'B Davies, ${ }^{2}$ Shepherd, ${ }^{3} \mathrm{D}$ Tuthill, ${ }^{3} \mathrm{Z}$ Roberts. 'School of Medicine, Cardiff University, Cardiff, UK; ${ }^{2}$ Violence Research Unit, Cardiff University, Cardiff, UK; ${ }^{3}$ The Children's Hospital for Wales, Cardiff, UK

\subsection{6/archdischild-2018-rcpch.332}

Background Community violence remains a significant and worrying element of society. This is especially true among children, where most cases are teenager-on-teenager violence occurring near schools. Emergency departments (EDs) can play a vital role in protecting victims from further harm by referring them to safeguarding agencies. A previous audit (2010) from the University Hospital of Wales' ED revealed only 3\% of paediatric community violence victims under the age of 16 were referred on.

Aim To review the ED's current response to children injured in community violence.

Methods Records were sourced using Cardiff Model data. ED records of children ( $<18$ years) who presented over 2 years as alleged community violence victims were retrospectively reviewed for documented referral to 'protection' agencies. The audit standard was that a referral should have been made to: the school nurse if the child was in education; social services if there was a Safeguarding concern, or they were already involved; or the police if an adult committed the assault or a weapon was used.

Results 291 children were victims of alleged assault. When the details were documented, the commonest assault was in the street (46.4\%), committed by a child (50.1\%), using fists/ hands $(44.3 \%)$ causing soft tissue injuries (51.2\%). Serious harm (fracture or hospital admission) occurred in 38 cases (13.1\%). Overall, 165 (56.7\%) children were referred to at least one protection agency - however it varied by age (table 1). Those not referred included assaults involving drug abuse, weapons and teenage pregnancies.

\begin{tabular}{|c|c|c|c|c|}
\hline $\begin{array}{l}\text { Age } \\
\text { range }\end{array}$ & $\begin{array}{l}\text { Number } \\
\text { of victims }\end{array}$ & $\begin{array}{l}\% \text { referred if } \\
\text { required by the } \\
\text { standard }\end{array}$ & & \\
\hline & & Social services & School nurses & Police \\
\hline $0-5$ & 1 & 100 & N/A & N/A \\
\hline $6-9$ & 7 & 100 & 71.4 & 66.7 \\
\hline 10-13 & 68 & 75.9 & 69.1 & 71.4 \\
\hline 14-15 & 88 & 76.5 & 62.1 & 50.0 \\
\hline 16-17 & 127 & 23.6 & $\begin{array}{l}6.3 \text { (but education status was } \\
\text { not consistently documented) }\end{array}$ & 34.6 \\
\hline
\end{tabular}

Conclusion Improvements in referrals of child violence victims have occurred, but some remain vulnerable to harm including further assaults and mental health conditions. Recommendations following this audit comprised a revived focus on documenting alleged assault details and referring incidents involving older children and weapons.

\section{G343(P) A NEW HOPE? ARE PREDNISOLONE'S DAYS NUMBERED AS THE FORCE AWAKENS TO DEXAMETHASONE AS THE STEROID OF CHOICE IN THE TREATMENT OF ACUTE ASTHMA IN CHILDREN}

R Wockenforth, J Grice, R Searle, J Scott. Paediatric Accident and Emergency, Alder Hey Children's Hospital, Liverpool, UK

\subsection{6/archdischild-2018-rcpch.333}

Aims The Aims of this report were to compare the level of care provided by our A and E department in relation to the standards from the 2016 BTS guidelines for the management of acute asthma. In addition we report the use of dexamethasone as our new first line choice of steroid.

Methods This report is an audit of the children presenting to our hospital during the period between 1 st November 2016 and 30th June 2017 with a coded diagnosis of Asthma. The data was collected retrospectively and included children between the ages of 2 and 16 years of age. We assessed time to triage, correlation of triage to actual BTS classification of asthma, length of stay, combination of therapies and follow up. The data was compared with the previous audit

Results 404 asthma presentations between the 01/11/16 and 31/06/17.

124 episodes (twice as many as the previous audit) resulted in admission (99 under $\mathrm{A}$ and $\mathrm{E}$ clinicians to our emergency decision unit (EDU) and 25 admitted to the medical team).

The correlation of triage colour and severity assessment on examination was approximately $93 \%$.

$2.4 \%$ of patients became sicker as a result of waiting longer than their triage recommended.

The average length of stay when admitted under the emergency team to the EDU was 6 hours and $58 \mathrm{~min}$. When admitted under the medical team, the average length of stay was 3 days 16 hours and $33 \mathrm{~min}$.

$94.4 \%$ of patients were treated correctly compared with the BTS guidelines.

EDU has decreased our medical admissions by $80 \%$

There were no admissions from our $\mathrm{A}$ and $\mathrm{E}$ department to ITU during the audit period

Within 6 months prednisolone was phased out and only patients with specific care plans were using it

Conclusions We are operating to a high standard adhering to the BTS guidelines

Dexamethasone is a well tolerated and cheaper alternative to prednisolone- exact figures for savings are being calculated.

Triage continues to be a point of excellence for this emergency department

Over half of the children seen in this audit cycle were given follow up in an asthma nurse specialist or consultant led clinic.

\section{G344(P) SKIN DEEP? A CASE OF PSEUDOCHROMHIDROSIS PRESENTING TO THE CHILDREN'S ASSESSMENT UNIT (CAU)}

R Kirk, A Gite. Lister Hospital, East and North Hentfordshire NHS Trust, Stevenage, UK

\subsection{6/archdischild-2018-rcpch.334}

\section{Introduction}

- Chromhidrosis is a rare disorder beginning in puberty, characterised by the production of coloured sweat from sweat glands containing an increased number of lipofuscin pigments. 